\title{
EXISTENCE AND UNIQUENESS OF ANALYTIC SOLUTIONS OF THE SHABAT EQUATION
}

\author{
EUGENIA N. PETROPOULOU
}

Received 30 May 2004

Sufficient conditions are given so that the initial value problem for the Shabat equation has a unique analytic solution, which, together with its first derivative, converges absolutely for $z \in \mathbb{C}:|z|<T, T>0$. Moreover, a bound of this solution is given. The sufficient conditions involve only the initial condition, the parameters of the equation, and T. Furthermore, from these conditions, one can obtain an upper bound for $T$. Our results are in consistence with some recently found results.

\section{Introduction and main results}

Consider the nonlinear functional differential equation

$$
\begin{gathered}
f^{\prime}(z)+q^{2} f^{\prime}(q z)+f^{2}(z)-q^{2} f^{2}(q z)=\mu, \\
f(0)=f_{0},
\end{gathered}
$$

where $q, \mu$, and $f_{0}$ are in general complex numbers. Equation (1.1) for $q=1 / k, 0<k<1$, and $\mu=1-\left(1 / k^{2}\right)$ was derived by Shabat [10] when he considered the similarity solution of the dressing dynamical system

$$
\left(f_{j}+f_{j+1}\right)_{x}=f_{j}^{2}-f_{j+1}^{2}+\lambda_{j}-\lambda_{j+1}, \quad j=0, \pm 1, \pm 2, \ldots,
$$

which is closely interconnected with the spectral theory of the linear Schrödinger equation

$$
\psi_{x x}+[q(x)+\lambda] \psi=0
$$

Equation (1.1) is studied for $|q|<1$, because if $|q|>1$, then (1.1) is equivalent with

$$
\Phi^{\prime}(w)+p^{2} \Phi^{\prime}(p w)+\Phi^{2}(w)-p^{2} \Phi^{2}(p w)=-\mu p^{2}
$$

after setting

$$
f(z)=-\Phi(p w), \quad q z=w, \quad p=\frac{1}{q} .
$$


In the present paper, we give sufficient conditions so that the initial value problem (1.1)-(1.2) have a unique analytic solution which, together with its first derivative, converges absolutely for $z \in \mathbb{C}:|z|<T, T>0$. Moreover a bound for this solution is given. In particular, our result is the following theorem.

Theorem 1.1. Assume that $|q|<1, T>0$, and

$$
|1+q| \cdot\left|f_{0}\right|+T|\mu|<\frac{(1-|q|)^{2}}{4 T\left(1+|q|^{2}\right)} .
$$

Then the initial value problem (1.1)-(1.2) has a unique bounded analytic solution of the form

$$
f(z)=\sum_{n=1}^{\infty} \overline{\left(F, e_{n}\right)}\left(\frac{z}{T}\right)^{n-1}, \quad z \in \mathbb{C},
$$

which, together with its first derivative, converges absolutely for $|z|<T, T>0$. Moreover the following bound holds:

$$
|f(z)|<\frac{1-|q|}{2 T\left(1+|q|^{2}\right)} .
$$

Remark 1.2. In the case $T=1$, the initial value problem (1.1)-(1.2) has a unique bounded solution of the form $f(z)=\sum_{n=1}^{\infty}\left(F, e_{n}\right) z^{n-1}$ which, together with its first derivative, belongs to the Banach space:

$$
H_{1}(\Delta)=\left\{f(z)=\sum_{n=1}^{\infty} \bar{a}_{n} z^{n-1} \text { analytic in } \Delta \text { and } \sum_{n=1}^{\infty}\left|a_{n}\right|<+\infty\right\},
$$

where $\Delta=\{z \in \mathbb{C}:|z|<1\}$.

Combining Theorem 1.1 and relations (1.5) and (1.6), we obtain the following corollary.

Corollary 1.3. Assume that $|q|>1$ and

$$
|q| \cdot|1+q| \cdot\left|f_{0}\right|+T|\mu|<\frac{(|q|-1)^{2}|q|^{2}}{4 T\left(1+|q|^{2}\right)}, \quad T>0 .
$$

Then the initial value problem (1.1)-(1.2) has a unique bounded analytic solution of the form

$$
f(z)=-\sum_{n=1}^{\infty} \overline{\left(\Phi, e_{n}\right)}\left(\frac{z}{T}\right)^{n-1}, \quad z \in \mathbb{C}
$$

which, together with its first derivative, converges absolutely for $|z|<(T /|q|)$. Moreover the following bound holds:

$$
|f(z)|<\frac{(|q|-1)|q|}{2 T\left(1+|q|^{2}\right)}
$$


Remark 1.4. For the original Shabat equation, which is (1.1) for $q=1 / k, 0<k<1$, and $\mu=1-\left(1 / k^{2}\right)$, the above corollary becomes as follows. "If

$$
(k+1)\left|f_{0}\right|+\left(1-k^{2}\right) T<\frac{(1-k)^{2}}{4 T\left(1+k^{2}\right)}, \quad T>0,
$$

the initial value problem (1.1)-(1.2) for $q=1 / k, 0<k<1$, and $\mu=1-\left(1 / k^{2}\right)$ has a unique bounded analytic solution of the form

$$
f(z)=-\sum_{n=1}^{\infty} \overline{\left(\Phi, e_{n}\right)}\left(\frac{z}{T}\right)^{n-1}, \quad z \in \mathbb{C}
$$

which, together with its first derivative, converges absolutely for $|z|<k T$. Moreover the following bound holds:

$$
|f(z)|<\frac{1-k}{2 T\left(1+k^{2}\right)}
$$

Remark 1.5. The coefficients $\left(F, e_{n}\right)$ of $(1.8)$ are uniquely determined by the recurrence relation:

$$
\begin{gathered}
\left(1+q^{n}\right) \overline{\left(F, e_{n}\right)}=\frac{T}{n-1}\left(q^{n}-1\right) \sum_{k=1}^{n-1} \overline{\left(F, e_{k}\right)} \cdot \overline{\left(F, e_{n-k}\right)}, \quad n \geq 3, \\
\overline{\left(F, e_{1}\right)}=f_{0}, \quad \frac{1+q^{2}}{T} \overline{\left(F, e_{2}\right)}=\mu+\left(q^{2}-1\right) \overline{f_{0}^{2}}
\end{gathered}
$$

which for $T=1$ is found to be, after some simple manipulations, the same with the recurrence relation found in [13].

Remark 1.6. The following upper bound for $T$ can easily be obtained from (1.7),

$$
T<\frac{\sqrt{\left(1+|q|^{2}\right)\left[|\mu| \cdot(1-|q|)^{2}+\left(1+|q|^{2}\right)|1+q|^{2} \cdot\left|f_{0}\right|^{2}\right]}-\left(1+|q|^{2}\right)|1+q| \cdot\left|f_{0}\right|}{2|\mu|\left(1+|q|^{2}\right)}
$$

for $\mu \neq 0$.

Remark 1.7. Analogous results can also be obtained if instead of $\mu$ in (1.1), we have a complex function $\mu(z)$ which should be such that $\mu(T x) \in H_{1}(\Delta)$, for $x \in \Delta$. In this case, $|\mu|$ in (1.7) should be replaced by $\|\mu(T x)\|_{H_{1}(\Delta)}$.

The analytic solutions of the initial value problem (1.1)-(1.2), for complex or real parameters $\left(q, \mu, f_{0}\right)$, have been studied in $[7,13]$. More precisely, it was proved in [13, pages 63-65] that there exists a unique solution of the form

$$
f(z)=\sum_{n=0}^{\infty} f_{n} z^{n}
$$


which converges in the disc $|z|<R_{q}$ for every fixed value of $q \in \mathbb{C},|q|<1$. For the radius of convergence $R_{q}$, the following estimate was given in the same paper:

$$
R_{q} \geq \frac{\pi}{2 \sqrt{\left|\alpha_{1}\right| \alpha}}, \quad \alpha \equiv \frac{1+|q|^{2}}{1-|q|^{2}}, \quad \alpha_{1}=\frac{\mu}{1+q^{2}} .
$$

In order to prove this result, S. Skorik and V. Spiridonov used Taylor series, which is a method not applicable when $|q|=1$, as they state. However, they were able to give exact solutions of (1.1) in the cases where $q^{3}=1$ and $q^{4}=1$.

Recently, it was proved in [7] that if $|q|=1$ and

$$
\left|1+q^{n+1}\right|>(2 n)^{-v}, \quad\left|1-q^{n}\right|>(2 n)^{-\nu}, \quad n=1,2, \ldots,
$$

for some positive constant $\nu$ depending on $q$ only, there exists a unique solution of the form (1.19) of the initial value problem (1.1)-(1.2) (for $q, \mu, f_{0} \in \mathbb{C}$ ), which converges for $|z|<\varrho / 2^{7 v+1}$, where

$$
\varrho= \begin{cases}\frac{1}{2\left|f_{0}\right|}, & \mu=0, \\ \frac{\pi-2 \arctan \left(\sqrt{2 /|\mu|} \cdot\left|f_{0}\right|\right)}{2 \sqrt{2|\mu|}}, & \mu \neq 0 .\end{cases}
$$

For the proof of this result Liu followed Siegel's approach [12]. It is mentioned in [7] that "when $q$ is on the unit circle but not a root of unity, the analysis of the convergence of (1.19) is nontrivial since the coefficient $1+q^{n+1}$ can be arbitrarily small and a straightforward estimation of the coefficient $f_{n}$ is not enough." It is also mentioned there that in order to prove the convergence of (1.19), good estimates of $\delta_{n}, n=1,2, \ldots$, are needed, where $\delta_{n+1}=\left(1 /\left|1+q^{n+2}\right|\right) \max _{0 \leq m \leq n} \delta_{m} \delta_{n-m}, n \geq 0, \delta_{0}=1$.

Finally in [6], the regular solutions of the initial value problem (1.1)-(1.2) for $q, \mu, f_{0} \in$ $\mathbb{R}$ were studied. The following were proved among other things.

(1) If $q \in(0,1)$, then the initial value problem (1.1)-(1.2) has one and only one solution in a neighborhood of the origin with open maximal interval of existence $\left(T_{\min }, T_{\max }\right)$, for which $T_{\max },-T_{\min } \geq\left(1-q^{2}\right) /\left(\left(1+q^{2}\right)\left|f_{0}\right|\right), \mu=0, f_{0} \neq 0$ [6, Theorem 9(1), page 12].

(2) If $q \in(-1,0)$, then the initial value problem (1.1)-(1.2) has one and only one continuously differentiable solution in a neighborhood of the origin with open maximal interval of existence $\left(T_{\min }, T_{\max }\right)$, for which $T_{\max }>0, T_{\min }<0[6$, Theorem 16(1), page 25].

Remark 1.8. (i) For $\mu, q$, and $f_{0} \in \mathbb{R}$, our interval of existence is $(-T, T)$, which is a subset of $\left(T_{\min }, T_{\max }\right)$. Thus we have $T_{\min } \leq-T<T \leq T_{\max }$. Therefore $T_{\max }>0$ and $T_{\min }<0$, which is consistent with the second result of [6] mentioned above.

(ii) For $\mu=0$ and $q \in(0,1)$, we obtain the following from (1.7):

$$
T<\frac{(1-q)^{2}}{4\left(1+q^{2}\right)(1+q)\left|f_{0}\right|} .
$$


It can be easily proved that

$$
\frac{(1-q)^{2}}{4\left(1+q^{2}\right)(1+q)\left|f_{0}\right|}<\frac{1-q^{2}}{\left(1+q^{2}\right)\left|f_{0}\right|}
$$

Thus we have

$$
T<\frac{(1-q)^{2}}{4\left(1+q^{2}\right)(1+q)\left|f_{0}\right|}<\frac{1-q^{2}}{\left(1+q^{2}\right)\left|f_{0}\right|} \leq T_{\max },
$$

which is consistent with what is already mentioned.

The method we use is a functional analytic method developed by Ifantis [3] for differential and functional differential equations and used also in $[8,9,11]$ for functional and functional differential equations. The basic idea of the method is the equivalent transformation of the functional differential equation under consideration into an operator equation. By use of this method and due to the space $H_{1}(\Delta)$ where we work, the convergence of the established solution (in $H_{1}(\Delta)$ ) of the functional differential equation under consideration is immediately proved. In this way, we avoid the use of the method of majorizing series which is often used for proving the convergence of series and which was also used in [7].

This functional analytic method is briefly presented in Section 2. The proof of our main result (Theorem 1.1) is given in Section 3.

\section{The functional analytic method}

Denote by $H$ an abstract separable Hilbert space over the complex field with the orthonormal base $\left\{e_{n}\right\}, n=1,2,3, \ldots$, and by $(\cdot, \cdot)$ and $\|\cdot\|$, the scalar product and the norm in $H$, respectively. Consider now those elements $f \in H$, which satisfy the condition $\sum_{n=1}^{\infty}\left|\left(f, e_{n}\right)\right|<+\infty$. These elements form a Banach space $H_{1}$ with norm $\|f\|_{1}=$ $\sum_{n=1}^{\infty}\left|\left(f, e_{n}\right)\right|$. We also define the shift operators $V$ and $V^{*}$ as follows:

$$
\begin{gathered}
V: V e_{n}=e_{n+1}, \quad n=1,2, \ldots, \\
V^{*}: V^{*} e_{n}=e_{n-1}, \quad n=2,3, \ldots, V^{*} e_{1}=0 .
\end{gathered}
$$

It is proved [2, page 3139] that the mapping

$$
\phi(z)=\left(f_{z}, f\right)=\sum_{n=1}^{\infty} \overline{\left(f, e_{n}\right)} z^{n-1}, \quad|z|<1,
$$

is a one-to-one mapping from $H_{1}$ onto $H_{1}(\Delta)$ which preserves the norm, where $f_{z}=$ $\sum_{n=1}^{\infty} z^{n-1} e_{n}, f_{0}=e_{1},|z|<1$ are the eigenelements of $V^{*}$, which form a complete system in $H$, in the sense that $\left(f_{z}, h\right)=0$, for all $z,|z|<1$, implies that $h=0$. The element $f \in H_{1}$ defined by (2.2) is called the abstract form of $\phi(z)$. In general, the abstract form of a function $G(\phi(z)): H_{1}(\Delta) \rightarrow H_{1}(\Delta)$ is a mapping $N(f): H_{1} \rightarrow H_{1}$ for which the following relation holds:

$$
G(\phi(z))=\left(f_{z}, N(f)\right), \quad|z|<1 .
$$


From (2.2), [3, pages 89, 92], [4, page 355], [5, page 387] we have the following.

(i) The abstract form of $f(q z)$ is the element $Q^{*} f \in H_{1}$, where $Q^{*}$ is the adjoint of the diagonal operator $Q e_{n}=q^{n-1} e_{n},|q| \leq 1, n=1,2, \ldots$.

(ii) The abstract form of $f^{\prime}(z)$ is the element $C_{0} V^{*} f$, where $C_{0}$ is the diagonal operator

$$
C_{0} e_{n}=n e_{n}, \quad n=1,2, \ldots,
$$

for which it was proved in [3, Proposition 2] that it has a selfadjoint extension with discrete spectrum, that is, the domain of $C_{0}$ can be extended to the range of the bounded diagonal operator $B_{0}$ :

$$
B_{0} e_{n}=\frac{1}{n} e_{n}, \quad n=1,2, \ldots
$$

In [3, Proposition 3], it was also proved that the range of $B_{0}$, that is, the definition domain of $C_{0}$, is isomorphic with the linear manifold of $H_{2}(\Delta)$ which consists of all functions $f(z)$ with $f^{\prime}(z) \in H_{2}(\Delta)$, where $H_{2}(\Delta)$ is the following Hilbert space of analytic functions:

$$
H_{2}(\Delta)=\left\{f(z)=\sum_{n=1}^{\infty} \bar{a}_{n} z^{n-1} \text { analytic in } \Delta \text { and } \sum_{n=1}^{\infty}\left|a_{n}\right|^{2}<+\infty\right\},
$$

where $\Delta=\{z \in \mathbb{C}:|z|<1\}$.

(iii) The abstract form of $[f(z)]^{2}$ is the element $f(V) f$, where

$$
f(V)=\sum_{n=1}^{\infty}\left(f, e_{n}\right) V^{n-1}, \quad \text { for which }\|f(V)\|_{1}=\|f\|_{1} .
$$

It was also proved in [4, page 355], [5, page 386] that the operator $N(f)$ is Frechét differentiable at every point $f \in B(0, R)=\left\{f \in H_{1}:\|f\|_{1}<R\right\}$.

Due to the above known results, we can easily prove that

(iv) the abstract form of $f^{\prime}(q z)$ is the element $(1 / \bar{q}) C_{0} V^{*} Q^{*} f$,

(v) the abstract form of $[f(q z)]^{2}$ is the element $Q^{*} f(V) f$.

\section{Proof of Theorem 1.1}

Proof. First of all we set $x=z / T, f(z)=f(x T)=F(x)$, and (1.1)-(1.2) becomes

$$
\begin{gathered}
\frac{1}{T} F^{\prime}(x)+\frac{q^{2}}{T} F^{\prime}(q x)+F^{2}(x)-q^{2} F^{2}(q x)=\mu, \\
F(0)=f_{0} .
\end{gathered}
$$

According to what is mentioned in Section 2, the abstract form of (3.1) in $H_{1}$ is

$$
\frac{1}{T} C_{0} V^{*} F+\frac{\bar{q}}{T} C_{0} V^{*} Q^{*} F+F(V) F-\bar{q}^{2} Q^{*} F(V) F=\bar{\mu} e_{1},
$$


where $F$ is the abstract form in $H_{1}$ of $F(x)$ and $F(V)$ is defined by (2.7). Equation (3.3) can also be written as

$$
\left(I+\bar{q} Q^{*}\right) F=T\left[\frac{(1+\bar{q}) \bar{f}_{0}}{T} e_{1}+\bar{\mu} e_{2}-V B_{0} F(V) F+q^{2} V B_{0} Q^{*} F(V) F\right],
$$

where $B_{0}$ is defined by (2.5).

Since $|q|<1$, it follows that $\left\|\bar{q} Q^{*}\right\|_{1}=|q|<1$. Thus $\left(I+\bar{q} Q^{*}\right)^{-1}$ exists, is uniquely determined on all $H_{1}$, and is bounded by

$$
\left\|\left(I+\bar{q} Q^{*}\right)^{-1}\right\|_{1} \leq \frac{1}{1-|q|}
$$

Thus from (3.4), we obtain

$$
F=T\left(I+\bar{q} Q^{*}\right)^{-1}\left[\frac{(1+\bar{q}) \bar{f}_{0}}{T} e_{1}+\bar{\mu} e_{2}-V B_{0} F(V) F+q^{2} V B_{0} Q^{*} F(V) F\right]=\phi_{1}(F) .
$$

We would like to apply to (3.6) the fixed point theorem of Earle and Hamilton [1] which states that if $g: X \rightarrow X$ is holomorphic, that is, its Fréchet derivative exists, and $g(X)$ lies strictly inside $X$, then $g$ has a unique fixed point in $X$, where $X$ is a bounded, connected, and open subset of a Banach space $B$. According to what is mentioned in Section 2, it is obvious that the mapping $\phi_{1}(F)$ is Frechét differentiable.

Let $\|F\|_{1} \leq R, R$ sufficiently large but finite. Then we find from (3.6)

$$
\begin{aligned}
\left\|\phi_{1}(F)\right\|_{1} & \leq \frac{T}{1-|q|}\left(\frac{|1+q| \cdot\left|f_{0}\right|}{T}+|\mu|+\|F\|_{1}^{2}+|q|^{2}\|F\|_{1}^{2}\right) \\
& \Longrightarrow\left\|\phi_{1}(F)\right\|_{1} \leq \frac{|1+q| \cdot\left|f_{0}\right|+T|\mu|}{1-|q|}+T \frac{1+|q|^{2}}{1-|q|} R^{2} .
\end{aligned}
$$

Let $P(R)=R-T\left(\left(1+|q|^{2}\right) /(1-|q|)\right) R^{2}$. This function has a maximum at the point $R_{0}=(1-|q|) /\left(2 T\left(1+|q|^{2}\right)\right)$ which is $P\left(R_{0}\right)=(1-|q|) /\left(4 T\left(1+|q|^{2}\right)\right)$. Then for $\|F\|_{1}<$ $R_{0}$ and for all $\epsilon>0$, if

$$
\frac{|1+q| \cdot\left|f_{0}\right|+T|\mu|}{1-|q|} \leq P\left(R_{0}\right)-\epsilon
$$

it follows from (3.7) that

$$
\left\|\phi_{1}(F)\right\|_{1} \leq R_{0}-\epsilon<R_{0}
$$

Thus if (1.7) holds, then due to the fixed point theorem of Earle and Hamilton, the operator equation (3.6) has a unique solution in $H_{1}$ bounded by $R_{0}$. Equivalently, the initial value problem (3.1)-(3.2) has a unique solution bounded by $R_{0}$ which, together with its first derivative, belongs to $H_{1}(\Delta)$. This means that the initial value problem (1.1)-(1.2) has a unique solution bounded by $R_{0}$ which, together with its first derivative, converges 
absolutely for $|z|<T$. The bound of $f$ is derived as follows:

$$
\begin{aligned}
|f(z)| & =|f(x T)|=|F(x)| \\
& \leq\|F(x)\|_{H_{1}(\Delta)}=\|F\|_{1}<R_{0} .
\end{aligned}
$$

Remark 3.1. The recurrence relation (1.17) is obtained by taking the inner product of both parts of (3.4) with $e_{n}$ and $e_{2}$.

\section{Acknowledgment}

This work was supported by a postdoctoral grant from the National Foundation of Scholarships (IKY).

\section{References}

[1] C. J. Earle and R. S. Hamilton, A fixed point theorem for holomorphic mappings, Global Analysis (Proc. Sympos. Pure Math., Vol. XVI, Berkeley, (1968), American Mathematical Society, Rhode Island, 1970, pp. 61-65.

[2] E. K. Ifantis, Structure of the point spectrum of Schrödinger-type tridiagonal operators, J. Mathematical Phys. 11 (1970), no. 11, 3138-3144.

[3] - An existence theory for functional-differential equations and functional-differential systems, J. Differential Equations 29 (1978), no. 1, 86-104.

[4] _ Analytic solutions for nonlinear differential equations, J. Math. Anal. Appl. 124 (1987), no. $2,339-380$.

[5]_, Global analytic solutions of the radial nonlinear wave equation, J. Math. Anal. Appl. 124 (1987), no. 2, 381-410.

[6] Y. Liu, Regular solutions of the Shabat equation, J. Differential Equations 154 (1999), no. 1, 141.

[7] An existence result for the Shabat equation, Aequationes Math. 64 (2002), no. 1-2, 104109.

[8] E. N. Petropoulou, Analytic solutions of a class of linear and nonlinear functional equations, J. Math. Anal. Appl. 261 (2001), no. 1, 168-176.

[9] E. N. Petropoulou and P. D. Siafarikas, Erratum: "Existence and uniqueness of solutions in $H_{1}(\Delta)$ of a general class of non-linear functional equations" [J. Math. Anal. Appl. 279 (2003), no. 2, 451-462], J. Math. Anal. Appl. 295 (2004), no. 1, 287-289.

[10] A. Shabat, The infinite-dimensional dressing dynamical system, Inverse Problems 8 (1992), no. 2, 303-308.

[11] P. D. Siafarikas, A singular functional-differential equation, Int. J. Math. Math. Sci. 5 (1982), no. 3, 497-501.

[12] C. L. Siegel, Iteration of analytic functions, Ann. of Math. (2) 43 (1942), no. 4, 607-612.

[13] S. Skorik and V. Spiridonov, Self-similar potentials and the q-oscillator algebra at roots of unity, Lett. Math. Phys. 28 (1993), no. 1, 59-74.

Eugenia N. Petropoulou: Division of Applied Mathematics and Mechanics, Department of Engineering Sciences, School of Engineering, University of Patras, 26500 Patras, Greece

E-mail address: jenpetro@des.upatras.gr 


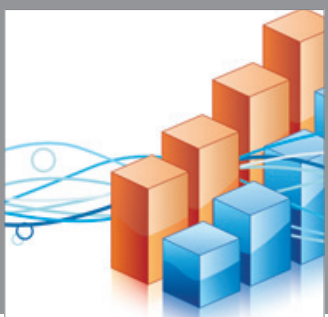

Advances in

Operations Research

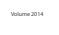

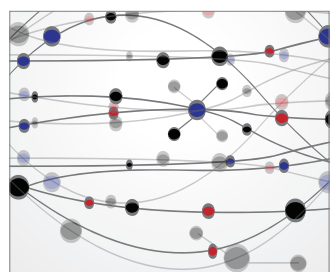

\section{The Scientific} World Journal
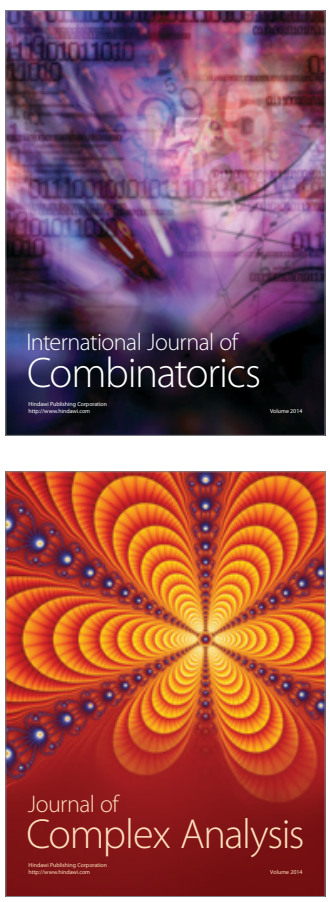

International Journal of

Mathematics and

Mathematical

Sciences
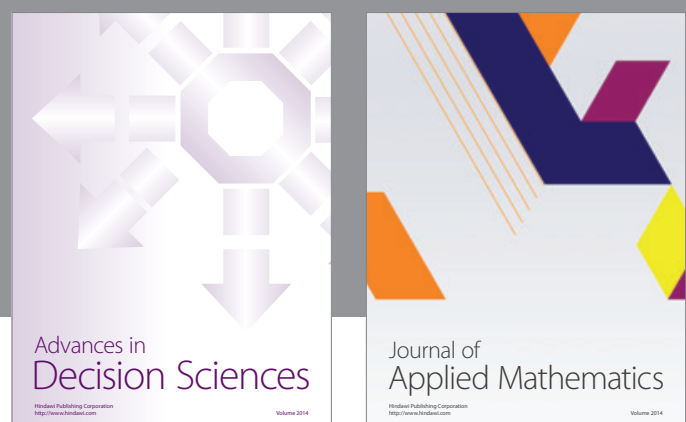

Journal of

Applied Mathematics
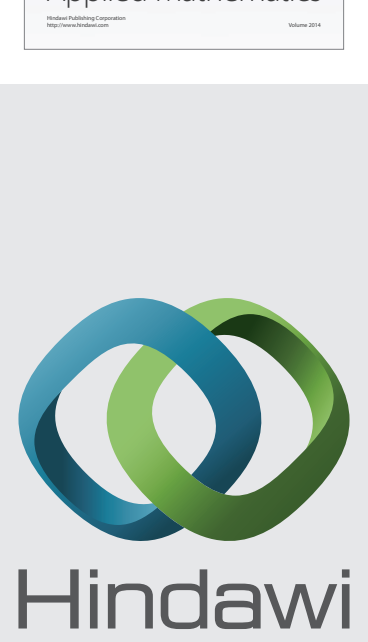

Submit your manuscripts at http://www.hindawi.com
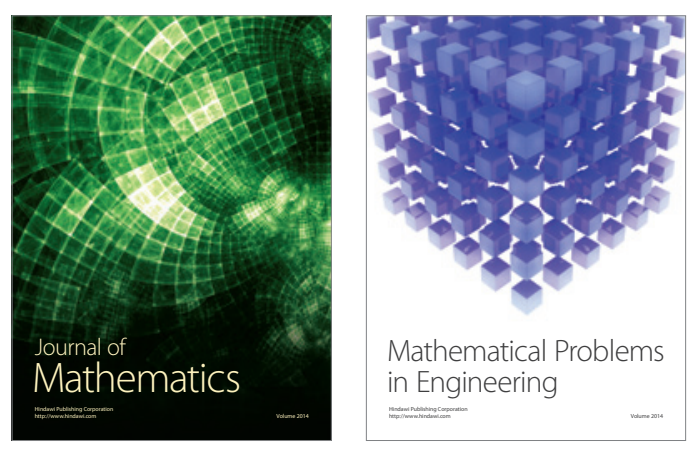

Mathematical Problems in Engineering
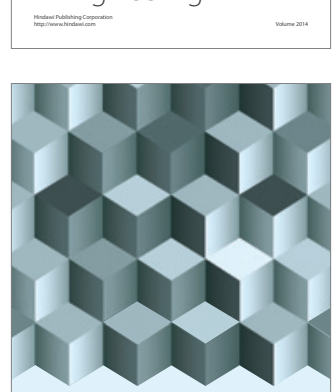

Journal of

Function Spaces
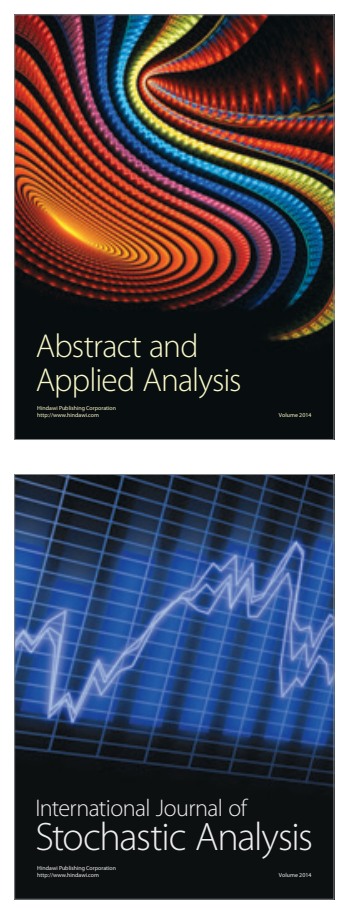

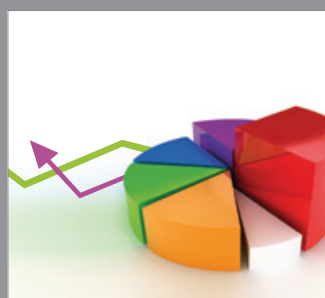

ournal of

Probability and Statistics

Promensencen
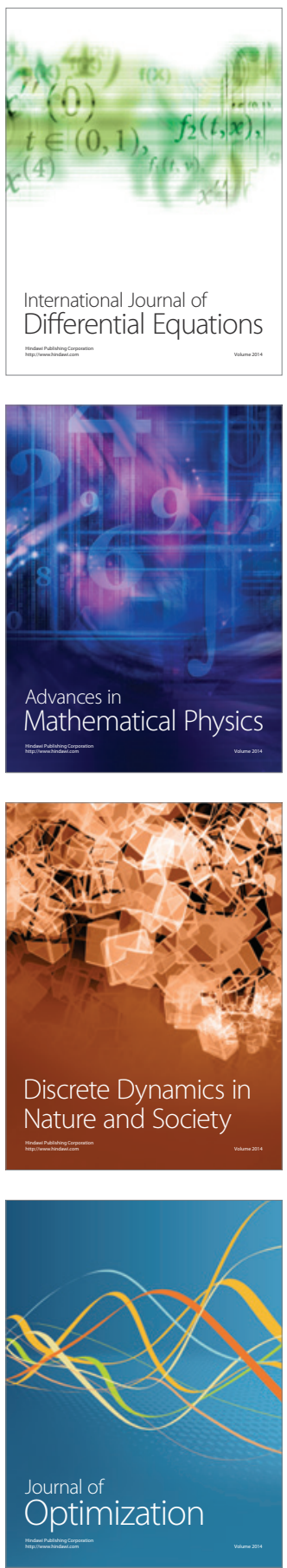